\title{
HUMANIZAÇÃO DO CUIDADO EM UNIDADE DE TERAPIA INTENSIVA: POTENCIALIDADES, DESAFIOS E ESTRATÉGIAS ${ }^{1}$
}

\author{
HUMANIZATION OF CARE IN AN INTENSIVE CARE UNIT: \\ POTENTIALITIES, CHALLENGES AND STRATEGIES
}

\author{
Natalya Garcêz Brill ${ }^{2}$, Rosiane Filipin Rangel ${ }^{3}$, Claudia Zamberlan ${ }^{4}$ e Silomar Ilha ${ }^{5}$
}

\section{RESUMO}

A Unidade de Terapia Intensiva (UTI) é o local destinado a pacientes graves e que possuem condições de recuperação, exigindo cuidado constante, algumas vezes, atrelado às técnicas e equipamentos, sendo necessário potencializar a humanização do cuidado. Dessa forma, esse estudo objetivou identificar as potencialidades, desafios/fragilidades vivenciados pela equipe de enfermagem no processo de humanização do em unidade de terapia intensiva, bem como as estratégias empregadas pelos mesmos, nesse processo. Trata-se de uma Revisão Narrativa da Literatura, realizada por meio de materiais on-line. Foi realizada, no mês de abril de 2018, uma busca nas bases de dados Literatura Latino Americana e do Caribe em Ciências de Saúde (LILACS) e Scieltific Eletronic Library Online (SCIELO), por meio dos descritores: Humanização da assistência, unidade de terapia intensiva e enfermagem. Os materiais selecionados foram submetidos técnica de Análise Textual Discursiva. Os resultados permitiram a identificação de potencialidades: a importância da humanização na UTI; a ambiência da UTI propícia à humanização; valorização da história de vida de cada paciente; importância da família no processo de humanização. Fragilidades: Sobrecarga de trabalho dos profissionais da UTI; déficit no processo de educação continuada; percepção da família como um empecilho para o bom andamento do trabalho dos profissionais da UTI; UTI vista como um ambiente mecânico e hostil. Estratégias: Cuidado/ assistência à família; e atividades de educação permanente com equipes de UTI. Conclui-se que as potencialidades, desafios/fragilidades e estratégias identificadas nesse estudo influenciam, direta ou indiretamente, no processo de humanização do cuidado na Unidade de Terapia Intensiva.

Palavras-chave: Humanização da assistência, Unidade de terapia intensiva, Enfermagem.

\section{ABSTRACT}

The Intensive Care Unit is the place for critically ill patients who are in conditions of recovery, requiring constant care, sometimes linked to techniques and equipment, and it is necessary to enhance the humanization of care. Thus, this study aimed to identify the potentialities, challenges/weaknesses experienced by the nursing

\footnotetext{
${ }^{1}$ Artigo derivado de Trabalho de Conclusão de curso de especialização

${ }^{2}$ Enfermeira. Especialista em terapia intensiva: ênfase em oncologia e controle de infecção hospitalar - Universidade Franciscana. Enfermeira assistencial na IMAX- Diagnóstico por Imagem. Santa Maria - RS, Brasil.

${ }^{3}$ Enfermeira. Docente do curso de enfermagem, pesquisadora júnior do mestrado profissional em saúde materno infantil e coordenadora do Programa de Residência Profissional em Enfermagem Obstétrica - Universidade Franciscana. Santa Maria - RS, Brasil. E-mail: E-mail: rosiane@ufn.edu.br

${ }^{4}$ Enfermeira. Docente do curso de enfermagem, mestrado profissional em saúde materno infantil e coordenadora do Programa de Residência Multiprofissional em Atenção clínica especializada com ênfase em infectologia e neurologia Universidade Franciscana. Santa Maria - RS, Brasil. E-mail: E-mail: claudiaz@ufn.edu.br

${ }^{5}$ Orientador. Enfermeiro. Docente do curso de enfermagem, pesquisador júnior do mestrado em ciências da saúde e da vida e coordenador do Programa de Residência Profissional em Enfermagem na Urgência/Trauma - Universidade Franciscana. Santa Maria - RS, Brasil. E-mail: silomar.ilha@unifra.br
} 
team in the humanization process of the Intensive Care Unit, as well as the strategies employed by them in this process. This is a Narrative Literature Review, carried out using online materials. In April 2018, a search was performed in the databases of Latin American and Caribbean Literature in Health Sciences (LILACS) and Scieltific Eletronic Library Online (SCIELO), using the descriptors: Humanization of assistance, therapy unit intensive care and nursing. The selected materials were submitted to the Discursive Textual Analysis technique. The results allowed the identification of potentialities: the importance of humanization in the ICU; the ICU ambience conducive to humanization; valuing the life history of each patient; importance of the family in the humanization process. Weaknesses: Work overload of ICU professionals; deficit in the continuing education process; perception of the family as an obstacle to the smooth running of the work of ICU professionals; ICU seen as a mechanical and hostile environment. Strategies: Care / assistance to the family; and, permanent education activities with ICU teams. It is concluded that the potentialities, challenges / weaknesses and strategies identified in this study, directly or indirectly influence the process of humanization of care in the Intensive Care Unit.

Keywords: Humanization of assistance, Intensive care units, Nursing.

\section{INTRODUÇÃO}

A Unidade de Terapia Intensiva (UTI) é um local destinado a pacientes graves, porém que possuam condições de recuperação, o que exige um cuidado constante e de qualidade a esses pacientes (SILVEIRA; CONTIM, 2015). A UTI foi criada devido a necessidade de aperfeiçoamento na assistência à pacientes graves ou potencialmente graves e conta com instrumentos tecnológicos e com $\mathrm{o}$ aprimoramento das habilidades dos profissionais para o atendimento adequado às necessidades de cuidados complexos (CAMPONOGARA et al., 2011).

Nessa perspectiva, a UTI conta com o suporte de diversos equipamentos para que a equipe de saúde possa prestar a assistência necessária a esses pacientes, sendo considerada uma unidade de internação especializada. Contudo, faz-se necessário um olhar voltado ao cuidado humanizado, pois devido a disposição de tecnologias e equipamentos neste ambiente, algumas vezes, percebe-se a preocupação mais voltada para técnicas e à realização do trabalho de forma mecanizada (FARIAS et al., 2013).

O ambiente da UTI propicia uma rotina diária e complexa, em que se insere o trabalho da equipe multidisciplinar na assistência ao paciente crítico, fazendo com que, por vezes, esses profissionais esqueçam de tocar, conversar e ouvir o ser humano que está a sua frente, o que torna o processo de humanização no cuidado em UTI uma tarefa difícil. Tal processo, suscita de atitudes sensíveis e singulares em um ambiente considerado altamente tecnológico (FARIAS et al., 2013).

A humanização é definida como um conjunto de ações que engloba não só o atendimento de qualidade através de um suporte tecnológico eficaz, mas também o cuidado ao paciente e seus familiares através do acolhimento efetivo e do respeito ético e cultural dos pacientes (SANCHES, et al., 2016). Dessa forma, compreende-se a importância do acesso à tecnologia como ferramenta importante no processo de recuperação de pacientes internados, principalmente no que diz respeito as UTIs, devido ao quadro clínico em que os mesmos se encontram, o que remete ao conhecimento e 
capacitação da equipe multiprofissional frente a este cenário. Contudo, tão importante quando essas capacidades, está a necessidade de exercício diário do cuidado humanizado (SANCHES et al., 2016).

Frente a essa realidade a Política Nacional de Humanização (PNH) foi lançada no ano de 2003, com o objetivo de instigar mudanças nos modos de gerir e cuidar dos profissionais de saúde. A PNH estimula a comunicação entre gestores, trabalhadores e usuários na busca de processos coletivos de trabalho e afeto, com vistas a romper com práticas desumanizadoras que inibem a autonomia e a corresponsabilidade dos profissionais de saúde em seu trabalho (BRASIL, 2013).

A humanização dentro da UTI, busca a realização de um cuidado por parte da equipe multiprofissional ao paciente em estado crítico que interligue a assistência no aporte tecnológico com o cuidado humanizado. Representa um conjunto de iniciativas que visam a produção de cuidados em saúde por meio da conciliação entre a tecnologia disponível, a promoção de acolhimento, respeito ético e cultural do paciente (MARQUES; SAUZA, 2010).

Em um ambiente complexo como a UTI, onde estão pacientes em situação de vulnerabilidade física, pelo seu quadro clínico; e emocional, pelo enfrentamento da situação em que se encontram, o cuidado humanizado se torna necessário. A humanização é considerada, nesse contexto, um importante elemento, pois possibilita a ação do cuidado da equipe de saúde de forma humanística direcionada ao paciente e à sua família (COSTA; FIGUEIREDO; SCHAURICH, 2009).

Dentre os profissionais que compõe a equipe multiprofissional na UTI, destacam-se os de enfermagem, representados por enfermeiros e técnicos de enfermagem, pois são os que permanecem maior tempo ao lado dos pacientes em situações críticas. Os enfermeiros são responsáveis pela Sistematização da Assistência de Enfermagem (SAE), terminologia utilizada para a descrição da atuação gerencial desse profissional. Trata-se de uma metodologia que organiza o trabalho profissional quanto ao método, pessoal e instrumentos, tornando possível o desenvolvimento do cuidado (COFEN, 2009).

Além de serem responsáveis pela gestão da UTI, os enfermeiros implementam o plano de cuidados singular para cada paciente, compreendido como Processo de Enfermagem (PE), o qual se desenvolve por meio de cinco etapas: avalição (histórico de enfermagem), diagnósticos de enfermagem, planejamento, implementação e avaliação de enfermagem (COFEN, 2009). Os técnicos de enfermagem são igualmente necessários e importantes no contexto da UTI, pois são eles que desenvolvem a maioria dos cuidados prescritos pelo enfermeiro aos pacientes, bem como a terapia medicamentosa prescrita pelo médico.

Assim, tornam-se necessárias pesquisas sobre a vivência dos profissionais de enfermagem atuantes em UTI sobre a Humanização do cuidado, motivo que justifica a necessidade e relevância deste estudo. Frente ao exposto, questiona-se: Quais as potencialidades e os desafios/fragilidades vivenciadas pela equipe de enfermagem no processo de humanização do cuidado em Unidade de Terapia Intensiva? Quais as estratégias utilizadas pelos profissionais de enfermagem para o processo de humanização do cuidado em Unidade de Terapia Intensiva? 
Na tentativa de responder aos questionamentos, este estudo objetivou identificar as potencialidades, desafios/fragilidades vivenciados pela equipe de enfermagem no processo de humanização do cuidado em Unidade de Terapia Intensiva, bem como as estratégias empregadas pelos mesmos, nesse processo.

\section{MATERIAL E MÉTODOS}

Trata-se de um estudo de Revisão Narrativa da Literatura (RNL), a qual se constitui como uma publicação ampla apropriada para descrever e discutir o desenvolvimento ou o "estado da arte" de um determinado assunto, sob o ponto de vista teórico ou contextual. As RNL constituem-se, basicamente, de análise da literatura publicada em livros, sites, artigos de revistas impressas e ou eletrônicas, vídeos, manuais ministeriais, políticas públicas, anais de eventos e tudo que possa contribuir para o primeiro contato com o objeto de estudo. A RNL não necessita indicar as fontes utilizadas, a metodologia para a busca das referências e nem os critérios utilizados na avaliação e seleção dos trabalhos consultados (BRUN et al., 2015). Contudo, optou-se por descrever algumas informações.

Para esse estudo, utilizou-se materiais disponibilizados em formato on-line. Para tanto foi realizado, no mês de abril de 2018, uma busca nas bases de dados Literatura Latino Americana e do Caribe em Ciências de Saúde (LILACS) e Scieltific Eletronic Library Online (SCIELO), por meio dos descritores: Humanização da assistência, unidade de terapia intensiva e enfermagem. Estipulou-se como critério de inclusão para os estudos: artigos de pesquisa, estudos de caso, nas línguas portuguesa, inglesa e espanhola, publicados entre os anos de 2013 a 2018 e que atendessem ao objetivo do estudo. Optou-se pelo recorte temporal de cinco anos, por representar um referencial com dados considerados recentes acerca da temática estudada. Excluíram-se os materiais que não permitiram acesso livre, os que não versaram sobre a humanização ou que não trouxeram a palavra UTI no título ou resumo.

Foram encontrados um total de 114 estudos, os quais tiveram os seus títulos e os resumos revisados e aqueles que atenderam aos critérios de inclusão foram selecionados para análise. Na LILACS, ao cruzar os descritores: "Humanização da assistência" and "unidades de terapia intensiva" and "enfermagem" foram encontrados 37 estudos, dos quais três não atenderam ao objetivo do estudo; vinte e nove, não se encaixaram no recorte temporal; e dois, eram revisões da literatura, sendo considerados três artigos para esse estudo. Com os descritores: "humanização da assistência" and "unidades de terapia intensiva" foram encontrados 53 artigos, dos quais três não atenderam aos objetivos do estudo; 37, não se enquadraram no recorte temporal; quatro, eram revisões ou estudos de reflexão; e um, não disponibilizava o texto completo, sendo considerados nessa busca 5 artigos.

Na Scielo, com os descritores: "humanização da assistência" and "unidades de terapia intensiva" and "enfermagem", foram encontrados 06 artigos, dos quais; três, já haviam sido encontrados na LILACS; e três, não se encaixam no corte temporal. Com os descritores "humanização da assistência" and "unidades de terapia intensiva" foram encontrados 15 artigos dos quais; um, já havia 
sido encontrado na LILACS; três, não atenderam aos objetivos do estudo; nove, não se enquadravam no recorte temporal, sendo considerados nessa busca dois artigos. Com base nesta ação, atenderam ao objetivo e se somaram ao Corpus desse estudo, 10 artigos (Quadro 1).

Quadro 1 - Relação dos estudos selecionados, com demonstração do título e ano.

\begin{tabular}{|l|c|}
\hline \multicolumn{1}{|c|}{ Título do artigo } & Ano \\
\hline Humanização na teoria e na prática: a construção do agir de uma equipe de enfermeiros. & 2013 \\
\hline Cuidado humanizado em UTI: desafios na visão dos profissionais de saúde. & 2013 \\
\hline Clínica do cuidado de enfermagem na terapia intensiva: aliança entre técnica, tecnologia e humanização. & 2013 \\
\hline Humanização: nós abraçamos esta ideia. & 2013 \\
\hline O acolhimento no cuidado à família numa unidade de terapia intensiva. & 2015 \\
\hline Compreensão fenomenológica de enfermeiros intensivistas à luz de Peterson e Zderad. & 2015 \\
\hline Concepções de humanização de profissionais em Unidades de Terapia Intensiva Neonatal. & 2015 \\
\hline Humanização em UTI: sentidos e significados sob a ótica da equipe de saúde. & 2016 \\
\hline Percepções de profissionais de saúde sobre a humanização em unidade de terapia intensiva adulto. & 2016 \\
\hline Sofrimento moral de trabalhadores de uma UTI pediátrica. & 2017 \\
\hline
\end{tabular}

Fonte: dados da investigação, 2018.

Os artigos que atenderam aos critérios de inclusão foram submetidos a análise textual discursiva, organizada a partir de uma sequência recursiva de três componentes: 1) Unitarização, onde o pesquisador examinou com intensidade e profundidade os textos em detalhes, fragmentando-os no sentido de atingir unidades de significado; 2) Momento em que buscou-se o estabelecimento de relações entre as unidades de base, combinando-as e classificando-as, resultando em um ou mais níveis de categorias de análise; 3) Comunicação, onde o pesquisador apresentou as compreensões atingidas a partir dos dois focos anteriores, resultando nos metatextos, que foram constituídos de descrição e interpretação dos fenômenos investigados (MORAES; GALIAZZI, 2011).

\section{RESULTADOS E DISCUSSÃO}

A análise dos materiais permitiu a construção de uma categoria central: equipe de enfermagem no processo de humanização do cuidado em terapia intensiva. A mesma foi unitarizada em três unidades: potencialidades vivenciadas pela enfermagem no processo de humanização do cuidado em unidade de terapia intensiva; Desafios/fragilidades vivenciadas pela enfermagem no processo de humanização do cuidado em unidade de terapia intensiva e Estratégias utilizadas pela enfermagem para o processo de humanização do cuidado em unidade de terapia intensiva. Tais unidades, conduziram a 10 categorias, conforme a figura 1 . Nesse artigo discute-se, de forma descritiva, cada categoria dentro da respectiva unidade de base. 
Figura 1 - Representação esquemática da relação entre as categorias e as subcategorias.

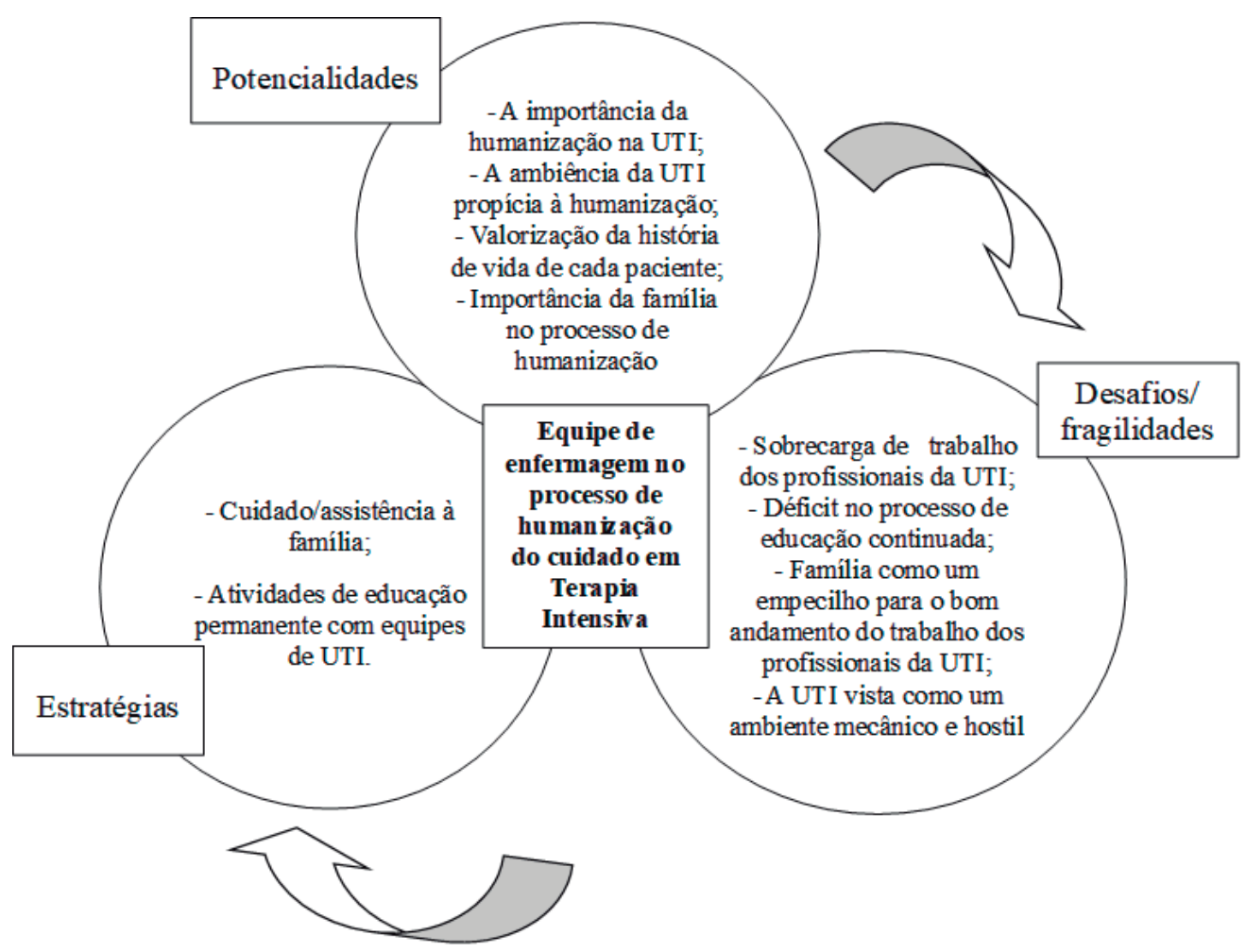

Fonte: elaboração própria, 2018.

\section{POTENCIALIDADES VIVENCIADAS PELA ENFERMAGEM NO PROCESSO DE HUMANIZAÇÃO DO CUIDADO EM UNIDADE DE TERAPIA INTENSIVA}

Algumas potencialidades vivenciadas pela equipe de enfermagem no que se refere ao processo de humanização do cuidado em UTI foram identificadas nos materiais utilizados, dentre elas destacaram-se, o conhecimento acerca da importância da humanização; a ambiência da UTI propícia à humanização; valorização da história de vida de cada paciente e o reconhecimento da importância da família no processo de humanização. Sobre o conhecimento acerca da importância da humanização, um artigo destacou que os entrevistados são conscientes da importância e dos benefícios do processo de humanização, pois tal processo auxilia no aspecto psicológico do paciente, estimulando-o a melhora e à redução do tempo de permanência na UTI (FARIAS et al., 2013).

O cuidado ao ser humano na UTI é realizado objetivando a sua qualidade de vida (QV) e bem estar, considerando o paciente na sua singularidade e complexidade (ROSEIRO; PAULA, 2015). Da mesma forma, o conceito de humanização é traduzido pelos profissionais entrevistados em um dos estudos analisados, como a busca pelo conforto do ser cuidado em todos os sentidos, oferecendo condições humanas no agir com bondade natural (SANCHES et al., 2016). Percebe-se dessa forma, que as concepções de humanização dos profissionais de saúde aproximam-se dos princípios norteadores da PNH, valorizando o ser humano como único, sem desconsiderar as suas relações interpessoais. 
Para tanto, denota-se que o cuidado humanizado é contrário ao cuidado restrito ao modelo tecnicista (ROSEIRO; PAULA, 2015).

Outra potencialidade percebida nos estudos versava acerca da UTI como um ambiente propício para a humanização. A esse respeito, um estudo fenomenológico realizado com sete enfermeiros que trabalhavam em uma UTI em Natal/RN, demonstrou que os enfermeiros consideravam a UTI um ambiente favorável para o desenvolvimento das ações de cuidado, pois possibilitava que a efetividade do trabalho humanizado fosse realizada por toda equipe (ARAÚJO; ARAÚJO, 2015). Corroborando com esse dado, estudo transversal realizado em quatro UTIs na cidade de São Paulo relatou que de forma geral, as UTI analisadas, possuíam ambientes de trabalho favoráveis ao exercício da prática profissional em enfermagem (BALSANELLI; CUNHA, 2013).

A valorização da história de vida de cada paciente, também apareceu nos materiais analisados como potencialidade. Em um estudo realizado com 23 profissionais de saúde em uma UTI, os participantes afirmam a necessidade de entender o indivíduo como ser único, com características individuais, com diferentes histórias de vida e diferentes formas de ver o mundo (MACHADO; SOARES, 2016). Ratificando este dado, um artigo demonstrou que a equipe de enfermagem buscava valorizar o paciente como um todo, considerando a visão do homem como ser não fragmentado, pela compreensão dos aspectos físicos, espirituais e sociais (OLIVEIRA et al., 2013).

Outra potencialidade encontrada nos materiais analisados, referia-se ao reconhecimento da importância da família no processo de internação na UTI. A esse respeito, estudo realizado com 29 profissionais de saúde, com o objetivo de investigar a concepção de humanização da equipe de profissionais de três UTIs Neonatal de Grande Vitória/ES, referiu que a presença da família foi um aspecto apontado pelos participantes como importante para oferta da assistência integral (ROSEIRO; PAULA, 2015).

Da mesma forma, em outro estudo os enfermeiros apontaram a necessidade de aproximação da família para que fosse possível realizar a assistência humanizada ao paciente, uma vez que a presença dos familiares é uma necessidade humana e precisa ser atendida (OLIVEIRA et al., 2013). Dessa forma, compreende-se que acolher a família do paciente, orientar e passar as informações sobre o seu estado de saúde é uma forma de continuidade do cuidado humanizado (PASSOS et al., 2015).

\section{DESAFIOS/FRAGILIDADES VIVENCIADAS PELA ENFERMAGEM NO PROCESSO DE HUMANIZAÇÃO DO CUIDADO EM UNIDADE DE TERAPIA INTENSIVA}

Um dos desafios/fragilidades que se destacou nos materiais analisados, versava acerca da sobrecarga de trabalho dos profissionais da UTI. Um artigo apresentou que a sobrecarga de trabalho do enfermeiro traz consequências negativas para o cuidado com o paciente, pois potencializa a execução de um cuidado restrito a técnica, realizado de forma mecânica, bem como o afastamento deste profissional em relação aos familiares (PASSOS et al., 2015). Outro estudo encontrou que as dificuldades de 
trabalho na UTI estavam relacionadas a alta carga horário, bem como a grande demanda de serviço (ROSEIRO; PAULA, 2015). O afastamento dos profissionais de saúde, no que diz respeito ao relacionamento com a família do paciente hospitalizado, é justificado em um artigo devido à sobrecarga de trabalho destes profissionais (OLIVEIRA et al., 2013).

Déficit no processo de educação continuada também foi evidenciado como um desafio/fragilidade nos estudos analisados. Uma pesquisa descritiva, exploratória de abordagem qualitativa, desenvolvida com 30 profissionais de enfermagem atuantes na UTI de um hospital público do município de Goiânia referiu-se a falta de interesse dos gestores acerca da necessidade de capacitação dos profissionais no que diz respeito a assistência humanizada, para que os mesmos se sentissem valorizados e motivados na realização do trabalho (OLIVEIRA et al., 2013).

A esse respeito, estudo desenvolvido com 20 profissionais de saúde, atuantes em uma UTI adulto demonstrou a necessidade do comprometimento por parte dos gestores para valorizar a equipe de saúde, fornecendo subsídios para que a mesma possa proporcionar um cuidado humanizado e acolhedor aos pacientes (FARIAS et al., 2013). Pode-se afirmar que programas de humanização no cuidado, ainda não eram implantados em inúmeras instituições de saúde, sendo necessário maior comprometimento por parte dos gestores na busca da realização dessas ações nestes cenários (MACHADO; SOARES, 2016).

Outro desafio/fragilidade versava acerca da percepção da família como um empecilho para o bom andamento do trabalho dos profissionais da UTI. Estudo desenvolvido com objetivo de descrever como o saber e o fazer (conceito e prática) humanização da assistência eram constituídos pelos enfermeiros em uma UTI pública de Goiânia, referiu que a assistência era dispensada a família do paciente, sendo a interação entre profissionais e familiares considerada superficial e ineficaz, uma vez que a presença da família era vista como empecilho para o bom andamento do trabalho realizado pelos profissionais na UTI (OLIVEIRA et al., 2013).

Nas falas de enfermeiras participantes de um estudo a respeito do acolhimento a família do paciente internado em UTI, se evidenciou o distanciamento da equipe com relação a interação com a família. No presente estudo, os profissionais referiram utilizar estratégias para que houvesse esse afastamento como, se ausentar da unidade para realizar as refeições no horário destinado a visita ou, permanecer em silêncio durante a prestação da assistência, resultando em um relacionamento distante com os familiares dos pacientes (PASSOS et al., 2015).

A UTI vista como um ambiente mecânico e hostil também foi evidenciado como um desafio/ fragilidade no processo de humanização. Tal dado, foi evidenciado em um estudo que analisou o sofrimento moral a que estavam expostos profissionais de saúde que atuavam na UTI pediátrica de um hospital no município do Sul do Brasil. Os resultados desse estudo, evidenciaram a despersonalização do cuidado criada pelo ambiente de trabalho (FACHINI; SCRIGNI; LIMA, 2017). Outro estudo corroborou com este achado, quando referiu que a UTI poderia se apresentar como um ambiente tenso 
e hostil, já que concentravam pacientes em estado grave que exigia atenção constante (CAETANO et al., 2007). Assim, se faz necessário, estimular a prática humanizada por parte dos profissionais com o objetivo de oferecer o melhor tratamento e cuidado possível.

Um estudo demonstrou que rotina diária no ambiente de UTI, o nível sensorial dos pacientes rebaixados, somado ao uso cotidiano de aparatos tecnológicos presentes neste ambiente contribuía, substancialmente, para que o cuidado se tornasse automático, o que dificultava a humanização da assistência ao paciente na percepção dos profissionais (SANCHES et al., 2016). O processo de humanização no cuidado ao paciente em UTI foi influenciado pelo aparato tecnológico amplamente difundido nos últimos anos, distanciando profissional, familiares e pacientes (SILVEIRA; CONTIM, 2015).

Compreende-se a contribuição e importância da tecnologia no que concerne o cuidado e tratamento dos pacientes, especialmente os internados no ambiente da UTI. Não se pretende, aqui, difundir a ideia erronia de que a tecnologia não deva ser utilizada ou que a mesma é negativa, mas sim que a sua utilização não substitui o toque humano, a avaliação clínica, o diálogo, a escuta e as relações entre profissional, paciente e familiares. Assim, defende-se que a tecnologia deve auxiliar no processo de cuidado e não ser ela própria, o cuidado.

\section{ESTRATÉGIAS UTILIZADAS PELA ENFERMAGEM PARA O PROCESSO DE HUMANI- ZAÇÃO DO CUIDADO EM UNIDADE DE TERAPIA INTENSIVA}

Como estratégia para auxiliar no processo de humanização na UTI destacou-se o cuidado/assistência à família. Um artigo que investigou o cuidado humanizado em UTI, identificou que os participantes utilizavam, como estratégia, o trabalho junto à família através do diálogo, da disponibilidade de horários para visita, aproveitando esses momentos para sanar suas dúvidas a respeito do paciente hospitalizado, considerando o familiar como um aliado no processo do cuidado humanizado (FARIAS et al., 2013). Outro estudo realizado com seis enfermeiras de uma UTI, apresentou como uma das estratégias no processo de humanização, o acolhimento dos familiares através do diálogo, buscando tranquilizá-los e orientá-los a respeito do estado de saúde e tratamento do paciente internado (PASSOS et al., 2015).

Também foram estratégias vivenciadas em uma UTI pediátrica de Minas Gerais, o acolhimento e cordialidade com os pais, a promoção de encontros com os pais para apoio e esclarecimentos, bom relacionamento com a família, o suporte emocional, além de proporcionar o cantinho dos pais, um ambiente de acolhimento à família (RODRIGUES; CALEGARI, 2016). Um estudo desenvolvido com 23 profissionais atuantes em uma UTI demonstrou que os profissionais reconheciam a disponibilização de informações aos pacientes e familiares, com ética, respeito ao próximo e empatia como processo de humanização no atendimento (MACHADO; SOARES, 2016).

A equipe de enfermagem, particularmente a que trabalha em UTI, desenvolve um cuidado contínuo aos pacientes internados, o que exige uma atenção integral por parte da mesma. Para tanto 
salienta-se a importância de o profissional exercer as suas atividades embasadas não somente na técnica, mas também em princípios éticos da profissão, o que permite refletir sobre o ser humano e suas ações (COSTA et al., 2014). Percebe-se que a equipe de enfermagem realiza o cuidado humanizado por meio da empatia, exercendo a comunicação terapêutica efetiva, ao passo que estabelece vínculos através do comprometimento com o cuidar (ARAÚJO; ARAÚJO, 2015).

Outra estratégia referida pelos profissionais de enfermagem em um dos estudos analisados, versava sobre as atividades de Educação Permanente (EP) com as equipes de UTI. Apesar do conhecimento acerca da importância do cuidado humanizado, como já fora visto anteriormente, tal forma de cuidado, algumas vezes não é realizado pela equipe de saúde devido a inúmeros fatores, entre eles: a sobrecarga de trabalho, os aparatos tecnológicos que se sobrepõe ao cuidado humanizado e os demais problemas cotidianos. Dessa forma torna-se evidente a necessidade da EP como uma ferramenta a ser utilizada pelo enfermeiro com o objetivo de estimular a equipe à realização da assistência humanizada, por meio da reflexão quanto as suas atitudes diante do cuidado no ambiente da UTI (MELLO et al., 2013).

Nessa perspectiva, o Ministério da Saúde propôs a Política Nacional de Educação Permanente em Saúde (PNEPS) como estratégia para a formação e o desenvolvimento de trabalhadores. A Educação Permanente em Saúde (EPS) é uma ferramenta que visa transformar e qualificar a atenção à saúde, os processos formativos, as práticas de educação em saúde, além de incentivar a organização das ações e dos serviços numa perspectiva intersetorial (BRASIL, 2009).

A EP apresenta papel importante no processo de reflexão da equipe de saúde, uma vez que possibilita a discussão acerca do conhecimento científico quanto ao processo do cuidado humanizado e da prática realizada no cotidiano dos profissionais (GODINHO; TAVARES, 2009). Além disso, se tona importante citar a necessidade da reflexão constate por parte da equipe de enfermagem, bem como dos demais profissionais da saúde, com relação a humanização no cuidado ao paciente hospitalizado, sendo que o processo de educação em saúde deve ser adequado de acordo com a realidade vivenciada pelos profissionais de saúde (SILVEIRA; CONTIM, 2015).

\section{CONSIDERAÇÕES FINAIS}

A realização desse estudo permitiu conhecer as potencialidades e os desafios/fragilidades vivenciados pela equipe de enfermagem no processo de humanização do cuidado em Unidade de Terapia Intensiva, bem como as estratégias utilizadas pelos profissionais de enfermagem para o processo de humanização do cuidado em Unidade de Terapia Intensiva.

Como principais potencialidades destacaram-se o conhecimento acerca da importância da humanização; a ambiência da UTI propícia à humanização; valorização da história de vida de cada paciente e o reconhecimento da importância da família no processo de humanização. Como fragilidades, 
a sobrecarga de trabalho dos profissionais da UTI; o déficit no processo de educação continuada; a percepção da família como um empecilho para o bom andamento do trabalho dos profissionais da UTI; e a UTI vista como um ambiente mecânico e hostil. Quanto as estratégias, salientaram-se o cuidado/assistência à família do paciente internado na UTI e a atividades de educação permanente com equipes de UTI.

Uma limitação do estudo, foi trabalhar somente com periódicos encontrados pela busca na Scielo e Lilacs, o que denota que outras publicações relevantes disponíveis em outras bases de dados, podem não ter sido captadas para análise. Contudo, essa revisão permitiu identificar, com ampla abrangência as questões que envolvem as potencialidades, desafios/fragilidades e estratégias que influenciam direta ou indiretamente no processo de humanização do cuidado na UTI.

Como contribuições desse estudo, entende-se que o mesmo pode ser considerado como diagnostico de alguns fatores que contribuem positivamente e/ou negativamente para o cuidado humanizado na realidade dos profissionais da UTI. Outrossim, esperasse que possa estimular os profissionais de diferentes áreas, em especial, os da enfermagem à construção de novas propostas e ampliação das estratégias com vistas a potencializar o processo de humanização do cuidado.

\section{REFERÊNCIAS}

ARAÚJO, L. M.; ARAÚJO, L. M. Compreensão fenomenológica de enfermeiros intensivistas à luz do pensamento humanístico de Paterson e Zderad. Revista de Enfermagem da Universidade do Estado do Rio de Janeiro, Rio de Janeiro, v. 23, n. 3, p. 395-400, 2015.

BALSANELLI, A. P.; CUNHA, I. C. K. O. O ambiente de trabalho em unidades de terapia intensiva privadas e públicas. Acta paulista de enfermagem, São Paulo, v. 26, n. 6, p. 561-568, 2013.

BRASIL. Ministério da Saúde. Política Nacional de Educação Permanente em Saúde/Ministério da Saúde, Secretaria de Gestão do Trabalho e da Educação na Saúde, Departamento de Gestão da Educação em Saúde. - Brasília: Ministério da Saúde, 2009. 64 p. Disponível em: https://bit.ly/2IdVwWU. Acesso em: jul. 2020.

BRASIL. Ministério da Saúde. Política Nacional de Humanização (PNH). 1. Ed, Brasília-DF: Ministério da Saúde, 2013. 16 p. Disponível em: https://bit.ly/32va9fi. Acesso em: jul. 2020

BRUM, C. N. et al. Revisão narrativa: aspectos conceituais e metodológicos na construção do conhecimento da enfermagem. In: COSTENARO, R.; LACERDA, M. R. Metodologia da pesquisa para a enfermagem e saúde. Porto Alegre: Moriá, 2015. p. 124-142. 
CAETANO, J. A. et al. Cuidado humanizado em terapia intensiva: um estudo reflexivo. Escola Anna Nery - Revista de Enfermagem, Rio de Janeiro, v. 11, n. 2, p. 325-330, 2007.

CAMPONOGARA, S. et al. O cuidado humanizado em unidade de terapia intensiva: uma revisão bibliografica. Revista de Enfermagem da Universidade Federal de Santa Maria, Santa Maria, v. 1, n. 1, p. 124-132, 2011.

CONSELHO FEDERAL DE ENFERMAGEM. Resolução No 358/2009. Portal do Cofen - Conselho Federal de Enfermagem: Brasília/DF, 2009.

COSTA, S. C.; FIGUEIREDO, M. R. B.; SCHUARICH, D. Humanização em Unidade de Terapia Intenciva Adulto (UTI): Compreenções da equipe de enfermagem. Interface - Comunicação, Saúde, Educação, Botucatu, v. 13, supl. 1, p. 571-580, 2009.

COSTA, D. V. S. et al. A ética no contexto do cuidado de enfermagem na unidade de terapia intensiva: revisão integrativa. Revista de Tendências da Enfermagem Profissional, Ceará, v. 6, p. 1-13, 2014.

FACHINI, J. S.; SCRIGNI, A.V.; LIMA, R. C. G. S. Sofrimento moral de trabalhadores de uma UTI pediátrica. Revista bioética, Brasilia, v. 25, n. 1, p. 111-122, 2017.

FARIAS, F. B. B. et al. Cuidado humanizado em UTI: desafios na visão dos profissionais de saúde. Revista de pesquisa: cuidado é fundamental, Rio de Janeiro, v. 5, n. 4, p. 635-642, 2013.

GODINHO, J. S. L.; TAVARES, C. M. M. A educação permanente em enfermagem na uti neonatal: pesquisa exploratória de campo. 2009. 97f. Dissertação (mestrado Profissional Enfermagem Assistencial) - Universidade Federal Fluminence, Niterói, 2009.

MACHADO, E.R.; SOARES, N.V. Humanização em UTI: sentidos e significados sob a ótica da equipe de saúde. Revista de Enfermagem do Centro Oeste Mineiro, Minas Gerais, v. 6, n. 3, p. 2342-2348, 2016.

MARQUES, I. R.; SOUZA, A. R. Tecnologia e humanização em ambientes intensivos. Revista Brasileira de Enfermagem, Brasilia, v. 63 n.1, p. 141-144, 2010.

MELLO, B.L.D. et al. Humanização: nós abraçamos esta ideia. Arquivos de Ciências da Saúde da UNIPAR, Umuarama, v. 17, n. 3, p. 187-191, 2013. 
MORAES, R.; GALIAZZI, M. C. Análise textual discursiva. 2. ed. Ijuí: Editora Unijuí, 2011. 224p.

OLIVEIRA, N. E. S. et al. Humanização na teoria e na prática: a construção do agir de uma equipe de enfermeiros. Revista Eletrônica de Enfermagem, Goiás, v. 15, n. 2, p. 334-343, 2013.

PASSOS, S. S. S. et al. O acolhimento no cuidado à família numa unidade de terapia intensiva. Revista de Enfermagem da Universidade do Estado do Rio de Janeiro, Rio de Janeiro, v. 23, n. 3, p. 368-374, 2015.

RODRIGUES, A. C.; CALEGARI, T. Humanização da assistência na unidade de terapia intensiva pediátrica: perspectiva da equipe de enfermagem. Revista Mineira de Enfermagem, Belo Horizonte, v. 20, p. e933, 2016.

ROSEIRO, C. P.; PAULA, K. M. P. Concepções de humanização de profissionais em Unidades de Terapia Intensiva Neonatal. Estudos de Psicologia, Campinas, v. 32, n. 1, p. 109-119, 2015.

SANCHES, R. C. N. et al. Percepções de profissionais de saúde sobre a humanização em unidade de terapia intensiva adulto. Escola Anna Nery - Revista de Enfermagem, Rio de Janeiro, v. 20, n. 1, p. 48-54, 2016.

SILVEIRA, R. E.; CONTIM, D. Educação em saúde e prática humanizada da enfermagem em unidades de terapia intensiva: estudo bibliométrco. Revista de pesquisa: cuidado é fundamental, Rio de Janeiro, v. 7, n. 1, p. 2113-2122, 2015. 
